\title{
CLOSED FORM EXPRESSIONS FOR MAXIMUM DELAY AND JITTER IN MULTIBEAM OPPORTUNISTIC BEAMFORMING
}

\author{
Nizar Zorba \\ Centre Tecnologic de Telecomunicacions de Catalunya \\ Barcelona, Spain \\ email: nizar.zorba@cttc.es
}

\author{
Ana I. Perez-Neira \\ Universitat Politecnica de Catalunya \\ Barcelona, Spain \\ email: anuska@gps.tsc.upc.edu
}

\begin{abstract}
Scheduling in a Broadcast (BC) channel based on partial Channel State Information at the Transmitter (CSIT) is carried out through a multibeam opportunistic beamforming technique. Within a more practical perspective, this paper first presents a transmission strategy where a minimum rate per user is required, which in a wireless fading scenario, can only be guaranteed under a certain system outage constraint. This minimum rate is demanded within a given time interval to satisfy maximum delay restrictions for the user application. Closed form expressions for maximum scheduling delay and maximum jitter are obtained, standing as possible Quality of Service (QoS) indicators for the system behaviour. The derived expressions are then tested via simulations in several transmission scenarios.
\end{abstract}

\section{INTRODUCTION}

The Opportunistic multiantenna schemes are attractive due to their high performance, and at the same time, low complexity design. But while maximal sum rate has typically been considered as the objective of multibeam opportunistic schemes [1], an alternative approach that focuses on the QoS of the served users is required for a system implementation. A potential measure of the system QoS is through the minimum rate per user, so that each served user is guaranteed a minimum Signal-toNoise-Interference-Ratio (SNIR), allowing it to properly decode its intended data with a predefined Packet Error Rate (PER).

Previous studies [2] have shown that the user satisfaction is insignificantly increased by a performance higher than the user demands, while on the other hand, if the provided resources fail to guarantee its requirements, the satisfaction drastically decreases. Thus, a good scheduling scheme is achieved through satisfying the minimum requirements for the available users in the system.

Furthermore, the wireless operators realize that some users can provide deficient channel conditions for communication, and delivering service to such users can be very expensive in term of system resources, driving down the whole system performance; so that if these users are dropped, the operator can offer better service to all the remaining users in the system. Based on this practical point of view, operators are more interested in probability of outage measures [3] rather than absolute QoS fulfillment, making all the commercial systems to fix a target probability of outage in the users QoS.

\footnotetext{
${ }^{0}$ This work was partially supported by the Catalan Govemment grant SGR2005-00996; by the Spanish Govemment project TEC2005-08122-C03.
}

Very few works deal with QoS in opportunistic schemes, and no one (to the best of our knowledge) has presented QoS for multibeam opportunistic systems, where providing QoS is a challenging aspect due to cross interference among the scheduled users. This paper first presents the outage concept in multibeam opportunistic schemes, which matches with their possible application to commercial systems. Then it formalizes the service distribution statistics to obtain closed form solutions for the maximum scheduling delay and minimum rate per user. This approach provides a tool to optimize the system performance both in terms of the awarded rate and the maximum scheduling delay, therefore adapting the system behaviour to meet the system requirement for all the applications.

Moreover, with the delay expressions at hand, the system maximum delay jitter can be also characterized. This is actually a hard restriction for the system performance, as several applications need a fixed rate for the packets entering the applications decoder, where all commercial systems make use of the playout buffers [4, Chp.6] to deal with the random channels nature. The remainder of this paper is organized as follows: while section II deals with the system model, in section III a review of the multibeam opportunistic procedure is presented. Section IV presents the system QoS performance under outage specifications and derives the corresponding maximum scheduling delay and jitter equations. The numerical results and simulations are in section $\mathrm{V}$, to end with the paper conclusions in section VI.

\section{SySTEM MODEL}

We focus on the $\mathrm{BC}$ channel where $N$ receivers, each one of them equipped with a single receiving antenna, are being served by a transmitter at the Base Station (BS) provided with $n_{t}$ transmitting antennas, and supposing that $N$ is greater than $n_{t}$. A multiantenna channel $\mathbf{h}_{\left[1 \times n_{t}\right]}$ is considered between each of the users and the BS where a quasi static block fading model is assumed, which keeps constant through the coherence time, and independently changes between consecutive time intervals with independent and identically distributed (i.i.d.) complex Gaussian entries $\sim \mathcal{N}(0,1)$. Let $\mathbf{x}(t)$ be the $n_{t} \times 1$ transmitted vector, while denote $y_{i}(t)$ as the $i^{\text {th }}$ user received signal given by

$$
y_{i}(t)=\mathbf{h}_{i}(t) \mathbf{x}(t)+z_{i}(t)
$$

where $z_{i}(t)$ is an additive i.i.d. complex noise component with zero mean and $E\left\{\left|z_{i}\right|^{2}\right\}=\sigma^{2}$. The transmitter delivers service to $n_{t}$ simultaneous users, so that the transmitted signal $\mathbf{x}(t)$ incloses the uncorrelated data symbols $s_{i}(t)$ to each one of the selected users with $E\left\{\left|s_{i}\right|^{2}\right\}=1$, where a total transmitted power constraint of $P_{t}=1$ is considered. For ease of notation, time index is dropped whenever possible. 


\section{Multibeam Opportunistic TRANSMission}

One of the main transmission techniques in multiuser scenarios is the multibeam opportunistic beamforming [1], where random orthogonal beams are generated at the BS to simultaneously serve more than one user. The beam generation follows an orthogonal manner to guarantee the lowest interference among the served users, so that the maximum of $n_{t}$ beams are generated. Within the acquisition step, each one of the users sequentially calculates the SNIR related to each beam, and feeds back the best value to the BS together with an integer indicating the index of the preferred beam. The BS scheduler chooses the user with the largest SNIR value for each one of the beams, enters the transmission stage and forwards every one of the selected users with its intended data, where no user can obtain more than one beam at a time.

This multibeam strategy achieves high system sum rate by serving several users at the same time, making the transmitted signal to enclose the data symbols for the $n_{t}$ selected users as

$$
\mathbf{x}=\sqrt{\frac{1}{n_{t}}} \sum_{m=1}^{n_{t}} \mathbf{b}_{m} s_{m}
$$

with $\mathbf{b}_{m}$ as the unit power beam assigned to the $m^{\text {th }}$ user.

This transmission scheme is characterized by its SNIR term due to the interference that each beam generates to its nonintended users, representing a major drawback of this system. The SNIR formulation for the $i^{\text {th }}$ user through the $m^{\text {th }}$ beam, with several transmitting orthogonal beams, is as

$$
S N I R_{i, m}=\frac{\frac{1}{n_{t}}\left|\mathbf{h}_{i} \mathbf{b}_{m}\right|^{2}}{\sigma^{2}+\sum_{u \neq m}^{n_{t}} \frac{1}{n_{t}}\left|\mathbf{h}_{i} \mathbf{b}_{u}\right|^{2}}
$$

where no receiver processing is present and assuming uniform power allocation among all the users. And as exposed in [1], the paper considers that all the scheduled users present the same distribution for the SNIR value.

\section{SYSTEM QOS PERFORMANCE}

Once the rate benefits of the multibeam opportunistic schemes have been stated in several works in the literature [1], it turns to be the time to analyze the QoS performance of the opportunistic beamforming. QoS can be characterized by several metrics or indicators based on the design objectives, so that QoS can be in terms of rate, reflecting the minimum required rate per user, or in terms of delay, showing the maximum delay that a user can tolerate. This paper considers both concepts of QoS, where the transmission scheme guarantees a minimum rate per user, which is presented by minimum SNIR restriction (snir th per each user in the system, and delivered to it within a given maximum time delay.

As this work looks for realistic objectives with the implementation of the opportunistic schemes, it further drives the opportunistic schemes towards a practical point of view, through adapting an alternative service policy, where a predefined probability of outage $\xi_{\text {out }}$ in the service rate is tolerated [3], as done in cellular GSM and UMTS systems, and expected in home networking standards for delay intolerant applications.
The paper defines two concepts for outage [5], where the first one is related to the access scheme answering to the question: when the $i^{\text {th }}$ user will be provided service? Subsection (A.) characterizes the user access process to the system service and obtains the expression for its access probability. If a user does not access the channel within its maximum allowed delay, it is declared as being in delay outage $\xi_{\text {access. }}$. The second outage source comes through the awarded rate to answer the question: Once the $i^{\text {th }}$ user is selected for transmission, would it receive a data rate below its requirements? Subsection (B.) derives the rate distribution for the selected user, and obtains the minimum guaranteed rate under an outage $\xi_{\text {rate }}$, where the two kinds of outages are designed to meet the global system outage $\xi_{\text {out }}$.

\section{A. Delay Outage}

This section identifies the exact maximum time slot when the user will be served through any of the $n_{t}$ generated beams at the BS.

Definition: Given $\bar{P}_{\text {access }}$ as the probability of success on each trial, the probability that $K$ independent trials are required to select an element $i$ from a group of $N$ i.i.d. elements, is defined as the Geometric Distribution [6], having a cumulative distribution function (cdf)

$$
V(K)=1-\left(1-\bar{P}_{\text {access }}\right)^{K}
$$

which states that with a probability $V$, the $i^{\text {th }}$ element is selected in the first $K$ trials.

This definition matches the users access in an opportunistic scheme, where each one of the independent users tries to access to each one of the generated beams, with $\bar{P}_{\text {access }}=\frac{n_{t}}{N}$. Therefore, the maximum number of time slots until the $i^{\text {th }}$ user is selected with a probability of outage $\xi_{\text {access }}=1-V$, is given by

$$
K=\frac{\log _{2}(1-V)}{\log _{2}\left(1-\bar{P}_{\text {access }}\right)}=\frac{\log _{2}\left(\xi_{\text {access }}\right)}{\log _{2}\left(1-n_{t} / N\right)}
$$

where the effect of higher number of active users $N$ and the number of serving beams $n_{t}$ is controlled ${ }^{1}$.

\section{B. Rate Outage}

The user access to the system has been calculated in the previous section, but even if the user is selected, it may receive a rate that does not satisfy its SNIR requirements snir ${ }^{\text {th }}$, therefore going into a rate failure and causing an outage to that user. The paper calculates the distribution of the serving rate, to characterize the rate outage under a predefined $\xi_{\text {rate }}$.

Based on the opportunistic scheme philosophy to deliver service to the users, the serving SNIR value is the maximum SNIR over the active users in the system, corresponding to each generated beam. Using the SNIR equation in (3) with $n_{t}$ transmitted beams, note that the numerator follows a Chi-square $\chi^{2}(2)$ distribution while the interference terms in the denominator are modeled as $\chi^{2}\left(2\left(n_{t}-1\right)\right)$, which allows to obtain the probability distribution function (pdf) as (similar pdf calculation is developed in [1])

\footnotetext{
${ }^{1}$ A study by [7] reports access results when a single user is selected, but its results are based on Markovian chains, that complicate any funther development on its results to the multiple user selection.
} 
The 18th Annual IEEE International Symposium on Personal, Indoor and Mobile Radio Communications (PIMRC'07)

$$
f(x)=\frac{e^{-\left(x \cdot n_{t} \sigma^{2}\right)}}{(1+x)^{n_{t}}}\left(n_{t} \sigma^{2}(1+x)+n_{t}-1\right)
$$

and the cdf is then formulated as

$$
F(x)=1-\frac{e^{-\left(x \cdot n_{t} \sigma^{2}\right)}}{(1+x)^{n_{t}-1}}
$$

and since the serving SNIR is the maximum over all the users' SNIR values, then the serving SNIR cdf is stated as

$$
F F(x)=(F(x))^{N}=\left[1-\frac{e^{-\left(x \cdot n_{t} \sigma^{2}\right)}}{(1+x)^{n_{t}-1}}\right]^{N}
$$

Therefore, considering the cdf of the serving rate, the minimum required rate snir $^{\text {th }}$ for each user is achievable with a predefined rate outage $\xi_{\text {rate }}$ as

$$
\xi_{\text {rate }}=\left[1-\frac{e^{-\left(\text {snir }^{t h} \cdot n_{t} \sigma^{2}\right)}}{\left(1+\text { snir }^{t h}\right)^{n_{t}-1}}\right]^{N}
$$

where the values of $s n i r^{\text {th }}$ and $\xi_{\text {rate }}$ can be optimized to meet any system objectives under the number of users $N$. Notice that the rate outage as seen from an information theory pointof-view, corresponds to the PER measure in networking theory, so that both terms can be used in a related manner [8] in a block fading scenario, as the PER states

$$
P E R= \begin{cases}0 & \text { if } S N I R \geq \text { snirth } \\ 1 & \text { if } S N I R<\text { snir }\end{cases}
$$

where the direct relation to $\xi_{\text {rate }}$ is shown. With further manipulations, previous expression reformulates as

$$
\log _{2}\left(1+s n i r^{t h}\right)=\frac{\log _{2}\left(\frac{1}{1-\sqrt[N]{\xi_{\text {rate }}}}\right)-\lambda s n i r^{t h} \cdot n_{t} \sigma^{2}}{n_{t}-1}
$$

that will be later used, and where $\lambda=\log _{2}(e)=1.4427$ is adopted. It shows the rate limits of the system, where considering a fixed $\xi_{\text {rate }}$ parameter, a large snirth value can make previous expression to be negative, indicating that high outage $\xi_{\text {rate }}$ is required to enable large snir $^{\text {th }}$ values.

\section{System Outage}

As previously explained, the opportunistic transmission process comes controlled by two different outage measures, but the total system performance has to be defined through a single parameter.

Notice that the two discussed kinds of outage are totally independent, as a user access to the channel happens when its SNIR is the maximum over all the other users with respect to a given beam, but being the user with largest SNIR does not guarantee that this SNIR is larger than a given threshold snir ${ }^{\text {th }}$. Therefore, the total outage $\xi_{\text {out }}$ is defined as

$$
\xi_{\text {out }}=1-\left(1-\xi_{\text {access }}\right) \cdot\left(1-\xi_{\text {rate }}\right)
$$

where the operator has another degree of freedom to optimize the system through both $\xi_{\text {access }}$ and $\xi_{\text {rate }}$, as the commercial outage requirement can be imposed on the $\xi_{\text {out }}$ global measure.

\section{Maximum Scheduling Delay}

Once the scenario outage has been defined, the maximum scheduling delay can be obtained, where the paper defines the delay as follows

Definition: Having a packet of length $W$ bits corresponding to the $i^{\text {th }}$ user, and waiting for transmission at the BS scheduler, the scheduling delay is defined as the maximum required time to make the packet to reach its destination.

Notice that this definition of delay incloses the delay resulting from the scheduling process (i.e. the opportunistic selection) together with the delay caused by the channel transmission (i.e. low data rate), therefore providing a general expression for delay in opportunistic schemes. ${ }^{2}$. The smaller transmission unit is a packet, so that even the whole packet is transmitted, it fully remains at the BS buffer or it is dropped.

From previous section, the maximum number of time slots to select a user under a predefined delay outage was obtained as $K$, while this access provides a minimum rate of $R=\log _{2}(1+$ snir ${ }^{\text {th }}$ ) under a known rate outage. Therefore, the maximum scheduling delay, under an outage $\xi_{\text {out }}$ is as

$$
\text { maximum scheduling delay }=K=\frac{\log _{2}\left(\xi_{\text {access }}\right)}{\log _{2}\left(1-n_{t} / N\right)}
$$

showing the effect of the optimization variables. It is convenient to present a numerical example to avoid misleading conclusions for the reader, so that in a scenario with $N=30$, $n_{t}=3, K=25, \sigma^{2}=1$ and snir $^{\text {th }}=0.5$ for each user, it results that $\xi_{\text {access }}=7.1 \%$ and $\xi_{\text {rate }}=4.3 \%$ are obtained, therefore each user is guaranteed a correct reception of its packet within a maximum delay of 25 slots and with a total outage of $\xi_{\text {out }}=11.0 \%$.

Notice that the maximum delay expression for multibeam opportunistic schemes has not been provided in the literature, due to the wireless propagation characteristics, but thanks to the considered outage scenario and the formulated cdf for rate and access processes, the maximum delay can now be characterized under a global $\xi_{\text {out }}$ measure.

In the opportunistic scheduling policy only $n_{t}$ users do have access to each slot, therefore a more pragmatic system performance metric for the opportunistic schemes, is the user throughput normalized over each slot. With a system bandwidth $B_{w}$ and $t_{s}$ as the time slot (assumed to match the channel coherence time), the minimum normalized throughput defined in bits/slot states as

$$
T=B_{w} t_{s} \frac{R}{K}=\frac{B_{w} t_{s} \log _{2}\left(1-n_{t} / N\right) \log _{2}\left(1+\text { snir }^{\text {th }}\right)}{\log _{2}\left(\xi_{\text {access }}\right)}
$$

and plugging the expressions in (10), the throughput is obtained in a closed form at the top of the this page with all the involved optimization parameters.

\footnotetext{
${ }^{2}$ Since this paper concems about the scheduling delay, which is the most important kind of delay in the opportunistic scheme due to its multiuser service policy, both the buffer management and source statistics are not addressed [9]
} 
The 18th Annual IEEE International Symposium on Personal, Indoor and Mobile Radio Communications (PIMRC'07)

$$
T=\frac{B_{w} t_{s} \log _{2}\left(1-n_{t} / N\right)\left[\log _{2}\left(\frac{1}{1-\sqrt[N]{\xi_{\text {rate }}}}\right)-\lambda \text { snir }^{t h} \cdot n_{t} \sigma^{2}\right]}{\left(n_{t}-1\right) \log _{2}\left(\xi_{\text {access }}\right)}
$$

\section{E. Maximum Scheduling Jitter}

Once the user access policy and the maximum scheduling delay are characterized, it is quite tractable to obtain an expression for the maximum jitter under a predefined system outage, where the jitter is defined as

Definition: Having two consecutive packets of length $W$ bits each, corresponding to the $i^{\text {th }}$ user, and waiting for transmission at the BS scheduler. If the first packet is received at time $t_{1}$ while the second packet at time $t_{2}$, then the maximum jitter is defined [10] as the maximum difference between $t_{1}$ and $t_{2}$ :

$$
\text { maximum jitter }=\max \left(t_{2}-t_{1}\right)=\text { maximum delay } \cdot t_{s}
$$

Actually, this is a conservative expression for jitter, as it provides the maximum jitter, but it is required to characterize and design the communication system [10]. Plugging the expression (12) into the jitter formulation, gives the closed form expression of the maximum scheduling jitter.

For voice application, the packets have to enter to the voice decoder in a sequentially synchronized process, so that the voice application seems to be continuous to the final user [4]. This is only obtained if the playout buffer is correctly designed, and without a specific jitter formulation as the one in (15), the viability of the opportunistic schemes in delay constrained applications can be questioned.

\section{Simulations AND Numerical Results}

The performance of the proposed scheme is presented by Monte Carlo simulations, where we consider a scenario with $n_{t}=2$ transmitting antennas in a cell with a variable number of active users. The transmitter runs a multibeam opportunistic beamforming technique where two orthogonal beams are set up. A total system bandwidth of $1 \mathrm{MHz}$ with $t_{s}=1 \mathrm{msec}$ are considered. A noise variance of $\sigma^{2}=1$ is also assumed.

First of all, the paper studies the derived outages to test their certainty by simulations, so that in figure (1) a scenario with 10 active users is considered, and where the resulting $K$ value is plotted for several values of $\xi_{\text {access }}$. To provide the reader with more insight on the relevance of the $K$ parameter, the fitting to the integers of $K$ is also shown in the figure, as $K$ can only take integer values in real systems. The plot also shows how the theoretical integer values perfectly match the simulated ones.

Regarding the rate outage that the opportunistic systems incur due to the fading channel characteristics, figure (2) plots the $\xi_{\text {rate }}$ parameter for different values of minimum rate. The results exhibit how a higher rate outage $\xi_{\text {rate }}$ is obtained when the user requirement increases, which is also evident from the mathematical formulation in (9). The simulations show how the theoretically obtained results perfectly match the simulated values. Two number of active users are considered in the cell, to also show the effect of the multiuser gain on the outage performance, as a higher number of available users, provides more freedom to the scheduler to choose a better set of users to meet its requirements, so that lower outage is expected.

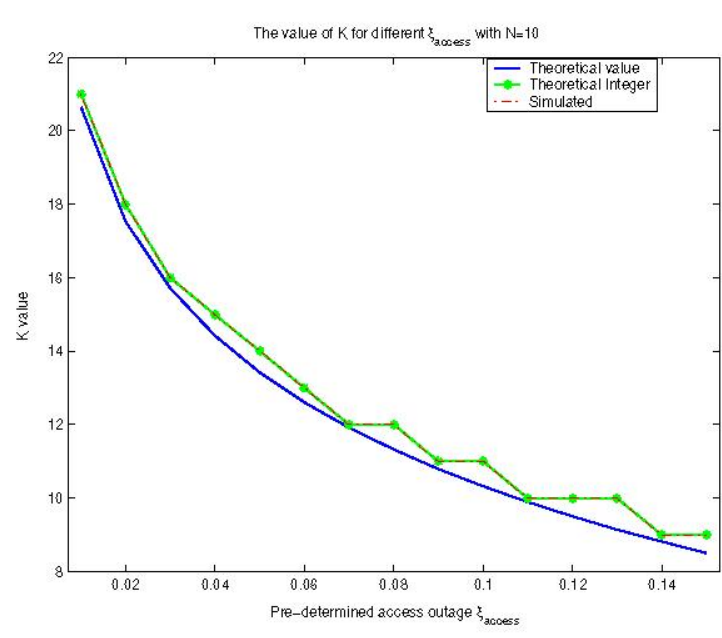

Figure 1: The system performance with access outage.

Concerned with the main motivation of this paper, the maximum scheduling delay performance is plotted in figure (3) for different values of outage $\xi_{\text {out }}$, where it is shown how the maximum delay is decreased for a larger allowed outage. Notice that a variable number of active users is available in the scenario, each one corresponding to a different outage value. A packet length of $W=160$ bytes is regarded in the simulations.

As already commented, a more pragmatic system metric in opportunistic schemes is the normalized throughput over the time slots, as a user is not serviced over all the time slots. Regarding the normalized system throughput in equation (13), figure (4) exhibits its results for different number of active users. It displays how a lower normalized throughput is obtained as more users are available in the system, as each serviced user has to wait for longer time until it is again supplied with service, thus decreasing its normalized throughput. The product between the normalized throughput and the waiting time $K$, certainly, provides the minimum rate with $\xi_{\text {access }}=10 \%$.

By considering a different scenario where the paper fixes a given total outage $\xi_{\text {out }}=0.20$ and tests for the maximum

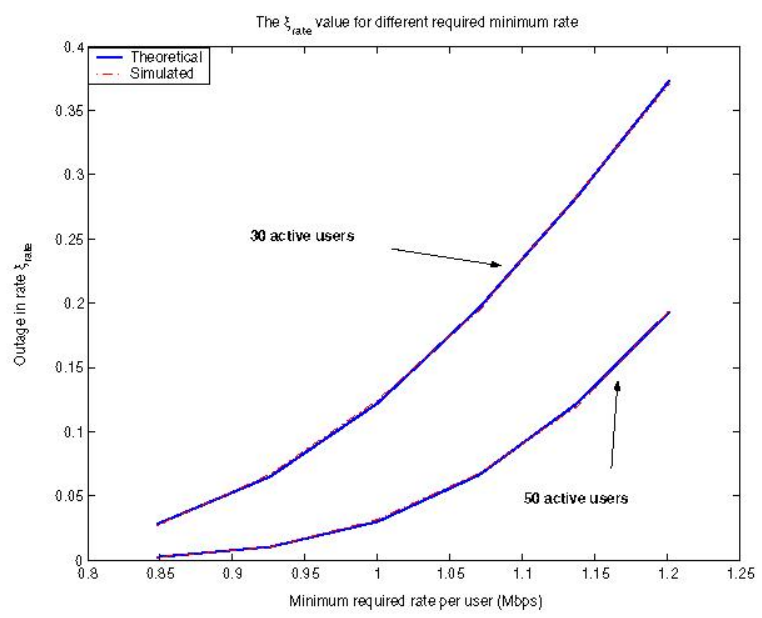

Figure 2: The system performance with rate outage. 


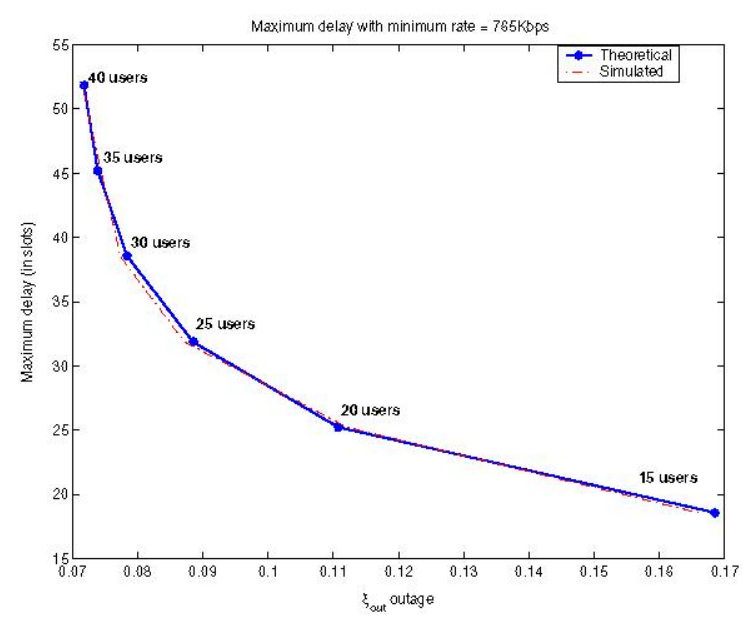

Figure 3: The system maximum scheduling delay in outage scenarios.

scheduling jitter performance when changing the number of users, but all of them under the same value of outage. The results are plot in figure (5), where an amazing result is obtained. Even it denotes the maximum jitter under outage performance, which is per-se an interesting result, but it also shows that there is an optimum number of users where each outage can be satisfied, with the minimum value for the maximum jitter. This is an interesting tool for network dimensioning as it indicates the optimal number of users in each cell for the best jitter performance. The obtained results are explained by the opportunistic transmission policy, where more users decrease the rate outage as previously seen in figure (2), but at the same time, a higher number of users means that each user has to wait for a longer time to have access to the network. The figure reflects that a large number of users is beneficial until a given point ( 24 users in the plot) due to the multiuser gain; but that a further increase in the number of users, does not compensate for their disadvantage in access. This last result motivates the need for an adaptive Call Admission Control (CAC) for its implementation in practical systems, which will be considered in future work.

\section{Summary ANd CONClusions}

Within a practical transmission system for commercial operators, a multibeam opportunistic scheme is set up with each

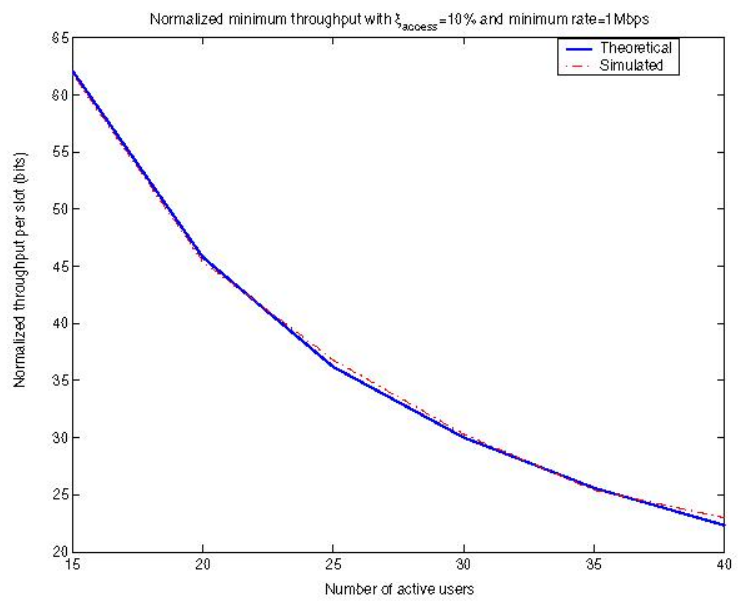

Figure 4: The normalized throughput per slot. user asking for a minimum rate to satisfy its QoS, where a predefined system outage is allowed. The outage comes from two sources, even through the users access process to the system resources, and/or due to the fading channel characteristics. The mathematical solutions for both cases are obtained, and a general outage measure is proposed.

Once the outage scenario statistics are obtained, the maximum scheduling delay that suffers a packet waiting for transmission is calculated, and given in a closed form solution. As a practical system indicator, the maximum system jitter is also stated for the considered scenario.

Simulations show the benefit of the obtained results for different environments, and the effect of the several optimization variables is studies, with a perfect match between the theoretically obtained equations and the simulated scenarios.

\section{REFERENCES}

[1] M. Sharif and B. Hassibi, "On the Capacity of MIMO Broadcast Channel with Partial Side Information," IEEE Trans. on Inform. Theory, vol. 51, no. 2, February 2005.

[2] N. Enderle and X. Lagrange, "User Satisfaction Models and Scheduling Algorithms for Packet-switched Services in UMTS," IEEE VTC-Spring, New Orleans-USA, 2003.

[3] N. Ahmed and R.G. Baraniuk, "Throughput measures for delayconstrained communications in fading channels," Allerton Conf., Monticello-USA, 2003.

[4] J.F. Kurose, and K.W. Ross, Computer Networking, Addison-Wesley Press, 2nd Edition, 2002.

[5] B. Hjelm, "Admission Control in Future Multi-Service Wideband DirectSequence CDMA Systems", IEEE VTC, Boston-USA, September 2000.

[6] M.R. Spiegel, Theory and Problems of Probability and Statistics. New York: McGraw-Hill, p. 118, 1992.

[7] I. Bettesh, and S. Shamai, "A low delay algorithm for the multiple access channel with Rayleigh fading", IEEE PIMRC, Massachusetts, 1998.

[8] T. Ghanim and M.C. Valenti, "The Throughput of Hybrid-ARQ in Block Fading under Modulation Constraints," CISS conference, Princeton, 2006.

[9] T. Issariyakul and E. Hossain, "Channel-Quality-Based Opportunistic Scheduling with ARQ in Multi-Rate Wireless Networks: Modeling and Analysis", IEEE Trans. Wireless Comm., vol. 5, no. 4, April 2006.

[10] C. Demichelis and P. Chimento, "IP Packet Delay Variation Metric for IP Perfomance Metrics (IPPM)", The Intemet Engineering Task Force, IETF-RFC, November 2002.

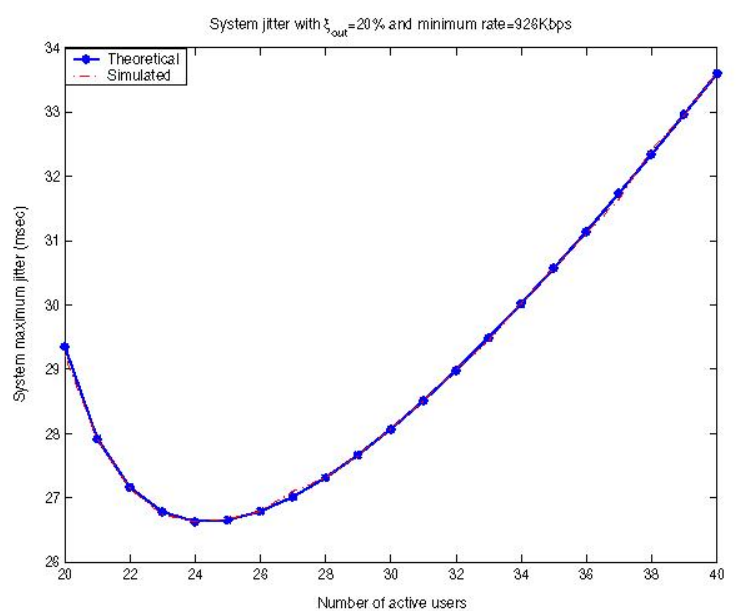

Figure 5: The system maximum jitter in outage scenarios. 